\title{
Die Parasitierung des Bohnenrostes Uromyces appendiculatus var. appendiculatus durch den Hyperparasiten Verticillium lecanii: Untersuchungen zur Wirt-Erkennung, Penetration und Abbau der Rostpilzsporen
}

\author{
Von \\ G. C. Grabski und K. Mendgen \\ Mit 2 Abbildungen \\ Eingegangen am 21. Dezember, 1984
}

\begin{abstract}
Hyperparasitism of Uromyces appendiculatus var. appendiculatus by Verticillium lecanii

Host Recognition, Penetration and Degradation of Spores
\end{abstract}

Culture filtrates of the hyperparasite Verticillium lecanii contain numerous lytic enzymes. When specific substrates were added to the filtrate, degradation of chitin is increased by a factor of 2,25 and degradation of starch is increased by a factor of 1,5. The degradation of uredo- and teliospores of Uromyces appendiculatus var. appendiculatus is documented cytologically. Appressorialike structures initiated direct penetration of the spore walls. Additional routes of penetration through the germpores of both sporetypes and the pedicles of the teliospores were observed. Degradation of the spore cy toplasm is described. Sugars on the surface of uredo- and teliospores and on hyphae of the hyperparasite were characterized using the gold-marked lectins Con A and WGA. Their role in the host-parasite recognition process is discussed.

\section{Zusammenfassung}

Der Hyperparasit V. lecanii verfügt über Enzyme, die in das Kulturmedium abgegeben werden. Bei Anwesenheit des spezifischen Substrates wird der Abbau von Chitin um den Faktor 2,25 und der Abbau von Stärke um den Faktor 1,5 gesteigert. Bei der Penetration von Uredo- und Teleutosporenwänden des Bohnenrostpilzes $U$. appendiculatus var. appendiculatus werden appressorienartige Strukturen ausgebildet, Infektionswege durch die Keimporen und den Teleutosporenstiel werden aufgezeigt. Der Abbau von Sporeninhalt und Sporenwänden beider Sporenformen des Bohnenrostes 
wird dokumentiert. Die an Goldpartikel gekoppelten Lektine Con A und WGA dienen dem Nachweis von Zuckerstrukturen auf den Sporenoberflächen des Bohnenrostes und den Hyphen des Hyperparasiten. Ihre Rolle zur Wirtserkennung wird diskutiert.

Der Deuteromycet V. lecanii parasitiert die Sporen der Rostpilze (MENDGEN und CASPER 1979, SPenCer 1980, HänSSler 1981, Mendgen 1981, Allen 1982). Bisherige Untersuchungen konzentrierten sich auf seine Biologie, die licht- und elektronenmikroskopische Darstellung der Penetration von Uredosporen und deren Abbau, sowie den möglichen Einsatz des Hyperparasiten im Gewächshaus (GRABSKI und MENDGEN 1985).

Die vorliegende Arbeit dokumentiert erstmals den Befall von Teleutosporen des Bohnenrostpilzes $U$. appendiculatus var. appendiculatus und deren Abbau durch Myzel des Hyperparasiten V. lecanii. Außerdem wurde die Wirt-Erkennung durch den Hyperparasiten untersucht, bei der die Ausscheidung von Enzymen und die Aktivierung der Enzymproduktion bei Anwesenheit des spezifischen Substrates eine größere Rolle zu spielen scheinen, als die Erkennung von Zuckerstrukturen, die auf den Sporenoberflächen mit Gold-markierten Lektinen nachgewiesen werden konnten.

\section{Material und Methoden}

\section{Pflanzenanzucht}

Die Buschbohne Phaseolus vulgaris var. Fori wurde in Töpfen mit Erde vom Typ ED 73 angezogen. Die jungen Sprößlinge durchbrachen nach 5 Tagen die Torfschicht, die Infektion mit Uredosporen des Bohnenrostpilzes erfolgte nach 15 Tagen.

\section{Anzucht von $U$. appendiculatus}

$0,33 \mathrm{mg} / \mathrm{ml}$ Uredosporen des Bohnenrostpilzes, von Dr. R. E. Gold von der Insel Reichenau isoliert (RBR) und freundlicherweise zur Verfügung gestellt, wurden in $0,05 \% \mathrm{v} / \mathrm{v}$ wäßriger Tween 20 Lösung aufgenommen und mit $0,2 \mathrm{mg} / \mathrm{ml}$ Talkum versetzt. Primärblätter 15 Tage alter Bohnen wurden mit dieser Suspension mittels Zerstäubers benebelt und 24 Std. in einer Feuchtekammer bei $100 \%$ rel. Luftfeuchte und Zimmertemperatur inkubiert. 10 Tage nach Infektion traten erste Uredolager auf, 25 Tage nach Infektion überwogen Teleutolager.

\section{Behandlung mit V. lecanii}

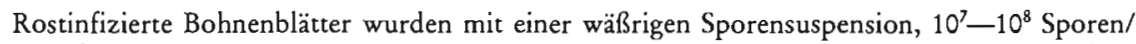
$\mathrm{ml}$, des V. lecanii-Isolates VK 1, das 1977 in Göttingen von Gelbrost, Puccinia striiformis, isoliert worden war, besprüht. Die Inkubation erfolgte in einer Feuchtekammer bei $100 \%$ rel. Luftfeuchte, $25^{\circ} \mathrm{C}$ und $10000 \mathrm{Lux}(16 \mathrm{Std} . / \mathrm{Tag}) .10$ Tage nach der Inokulation waren die Pusteln des Rostpilzes mit Myzel des Hyperparasiten überwachsen.

\section{Präparation zur Elektronenmikroskopie}

Proben wurden in $2 \%$ v/v Glutaraldehyd, $2 \%$ v/v Acrolein und $2 \%$ v/v Osmium (VIII) oxid fixiert, in 0,05 M Kalium-Phosphat-Puffer, $\mathrm{pH} 7,2$, gewaschen und in Alkohol entwässert. Die Infiltration in Ultra-Low-Viscosity-Harz (Fa. Polaron) wurde über eine Zwischenstufe mit Propylenoxid vorbereitet. Die Polymerisation erfolgte bei $70^{\circ} \mathrm{C}$ in $8 \mathrm{Std}$. 
Kupfergrids wurden mit einem Film aus 0,4\% w/v Pioloform in Chloroform belegt, der durch Auftragen von $10 \mathrm{~nm}$ Kohle in einer Balzers-Sputteranlage stabilisiert wurde.

Ultradünnschnitte wurden in $3 \% \mathrm{w} / \mathrm{v}$ wäßriger Uranylacetatlösung und Bleicitratlösung (REY NOLDS 1963) kontrastiert und in einem Siemens IA Elmiskop ausgewertet.

Bei der Präparation für die Rasterelektronenmikroskopie entfiel die Fixierung mit Acrolein und Osmium, die Proben wurden in 2 Schritten in Aceton überführt und durch Austausch des Acetons mit flüssigem Kohlendioxid in einer Kritisch-Punkt-Trocknungsanlage (Fa. Balzers) getrocknet. Nach Aufdampfen von $10 \mathrm{~nm}$ Gold und $10 \mathrm{~nm}$ Kohle erfolgte die Auswertung in einem "Cambridge Stereoscan“ (Limnologisches Institut, Universität Konstanz) oder einem Siemens Etec Rasterelektronenmikroskop (Institut für Virusforschung, MPI Tübingen).

\section{Präparation zur Untersuchung von Zelloberflächen}

Die Herstellung des Gold-Sols, die der Lektin-Gold-Komplexe, sowie die Kopplung der Lektin-Gold-Komplexe an die Proben, erfolgte nach bereits beschriebenen Methoden (Frens 1973, HORISBERGER et al. 1977 und 1979). Kontrollexperimente wurden durch Zumischung der spezifischen inhibitorischen Zucker: Methyl- $\alpha$-D-pyranosid ( $5 \mathrm{mg} / \mathrm{ml}$ ) für Con A und N-Acetyl-chito-pentaose $(2 \mathrm{mg} / \mathrm{ml})$ für WGA, durchgeführt. WGA wurde zur besseren Stabilisierung an Rinderserumalbumin (RSA) gekoppelt.

\section{Untersuchungen zur Exoenzymproduktion von V. lecanii}

Die Aktivität von chitinolytischen Enzymen, Amylasen, Lipasen und Proteasen wurde qualitativ auf Agarplatten bestimmt (HANKIN et al. 1975). Zusätzlich wurde die Induktivität der Enzymaktivitäten im Kulturfiltrat des Hyperparasiten durch die colorimetrische Bestimmung der freigesetzten Zuckermoleküle bestimmt (ELAD et al. 1983, Rerssig et al. 1955), indem V. lecanii in Schüttelkulturen mit Minimalmedium (DAvis et al. 1958) gezogen wurde, dem jeweils $4 \mathrm{~g} / \mathrm{L}$ des zu prüfenden CSubstrates beigefügt war. Die Kontrollkultur wurde in Glucose gezogen. Gemessen wurde gegen aqua dest., freie Zucker in den Kulturfiltraten wurden als Leerwerte in die Auswertung mit einbezogen.

\section{Ergebnisse}

\section{Elektronenmikroskopie}

Die Penetration von Uredosporen (Abb. 1 a: nicht infizierte Kontrolle) mit Hyphen des Hyperparasiten $V$. lecanii erfolgte meist auf der Sporenoberfläche (Abb. 1 b) nach Ausbildung appressorienartiger, polymorpher Strukturen oder direkt durch den Keimporus (Abb. $1 \mathrm{c}$ ). Wenn der Pilz bereits in die Spore eingedrungen war, konnte er auch wieder aus der Spore herauswachsen und die Sporenwand von innen nach außen durchbohren (Abb. $1 \mathrm{~d}$ ).

Penetrationsstellen in der Sporenwand hatten einen Durchmesser von $0,4 \mu \mathrm{m}$, der Durchmesser von Verticilliumhyphen dagegen betrug 0,8 $\mu \mathrm{m}$. Die Ränder von Penetrationsstellen waren glatt und wiesen keine Besonderheiten auf. Septierungen des Myzels vor der Penetration wurden häufig beobachtet.

Teleutosporen des Bohnenrostpilzes (Abb. 2 a: nicht infizierte Kontrolle) wurden über die Keimpore (Abb. 2 b, 2 f), den Stiel (Abb. 2 c) oder über die Sporenwand (Abb. 2 d, 2 e) infiziert, wobei in letzterem Fall appressorienartige Strukturen ausgeprägt wurden, die nach Verzweigung und Septierung des hyperparasitären Myzels auftraten (Abb. 2 e).

Zellinhalte von Uredo- und Teleutosporen wurden vollständig abgebaut. Querschnitte durch die Zellwände der Sporen zeigten, daß nicht immer der kürzeste Weg in das Sporeninnere gewählt wurde, sondern daß auch laterale Penetrationen vorkamen. 

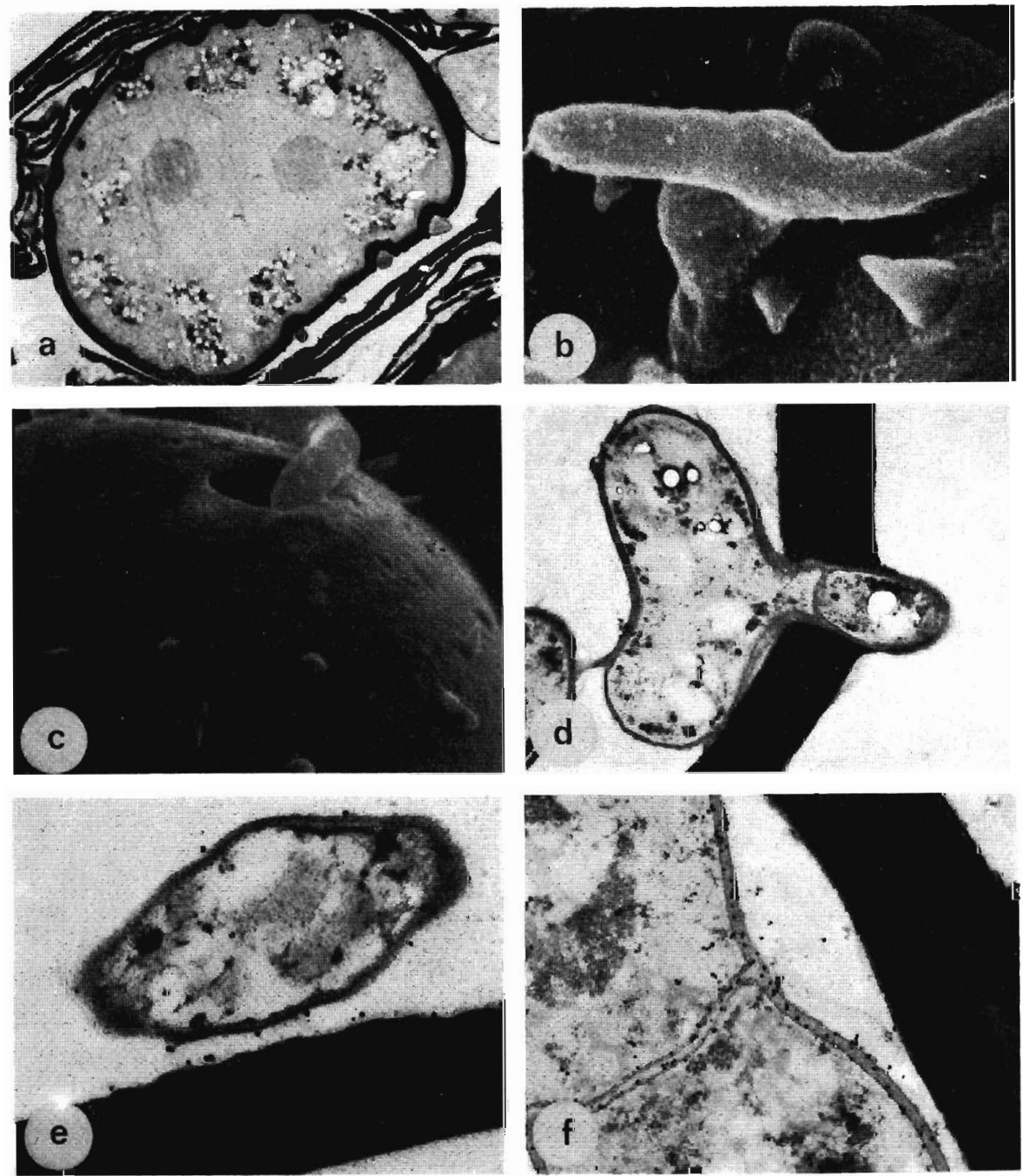

Abb. 1. Infektion von Uredosporen. a: nicht infizierte Uredospore, $\times 2500$, b: Infektion durch die Sporenwand nach Ausbildung eines Appressoriums, $\times 10350$, c: Infektion über den Keimporus, $\times$ 5400 , d: Penetration durch die Wand von innen nach außen, $\times 8000$, e: Kopplung von Goldmarkiertem Con A, $\times 15000$, f: Kopplung im post-embedding-Verfahren mit Gold-markiertem WGA-RSA, $\times 17000$

\section{Nachweis von Zuckerstrukturen}

Der Nachweis von Zuckermolekülen an der Oberfläche von Uredo- und Teleutosporen des Bohnenrostes gelang mit Gold markierten Lektinen: Concanavalin A (Con A), das an Glucose und/oder Mannose bindet und Wheat-GermAgglutinin (WGA), das an NAc-Glucosamin bindet. In allen Fällen wurde eine gleichmäßige Verteilung der Gold-Lektin-Komplexe an den Oberflächen der Bohnenrostsporen festgestellt (Abb. 1 e). Zusätzlich markiert wurden auch die 

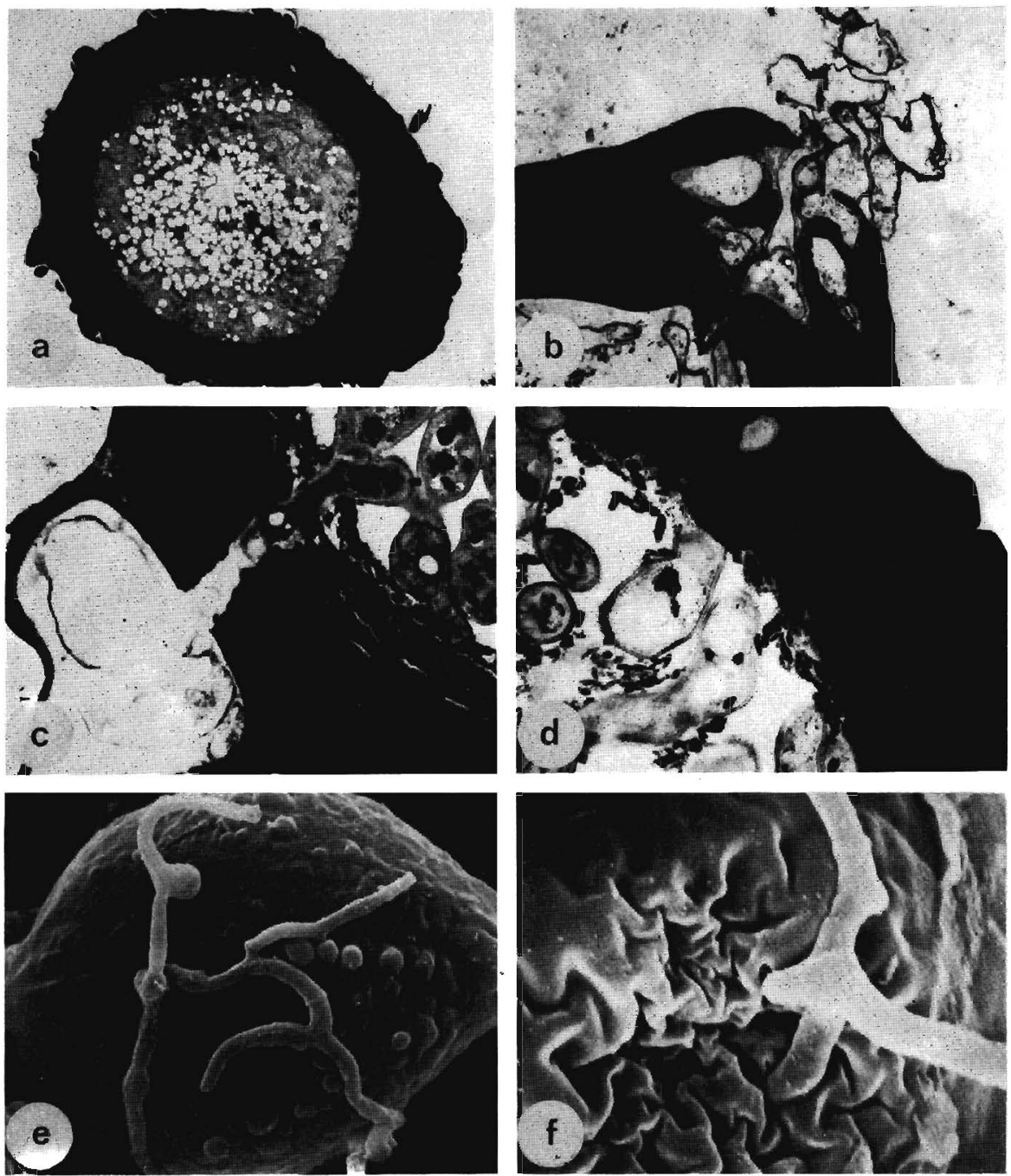

Abb. 2. Infektion von Teleutosporen. a: nicht infizierte Teleutospore, $\times 2500, b+c$ : Darstellung des Infektionsweges über die Keimpore, $\times 2800$ und über den Stiel, $\times$ 5500, d: Abbau der Sporenwand und Wanddurchbruch, $\times 8000$, e: Infektion über die Sporenwand, $\times 1824$, f: Infektion über die Keimpore, $\times 4800$

Hyphen des Hyperparasiten. Zellwände der Rostsporen und des Hyperparasiten, sowie Zellinhalt, wurden auch nach der Einbettung im Ultradünnschnitt mit WGA-RSA markiert (Abb. $1 \mathrm{f}$ ).

\section{Exoenzymproduktion des Hyperparasiten}

Durch die Bestimmung von Abbauzonen auf Agarplatten und durch colorimetrische Verfahren wurde festgestellt, daß der Hyperparasit $V$. lecanii Chitin, Tributyrin, Stärke, Cellulose, Laminarin und Protein mit in das Kulturmedium 
ausgeschiedenen Enzymen abbaut (Tabelle 1, 2). Die Induktion der Aktivität von chitinolytischen Enzymen und Amylase erfolgte nach Austausch von Glucose durch das jeweilige C-Substrat. Sie betrug bei Chitin $225 \%$ und bei Stärke $155 \%$, bezogen auf Vergleichswerte der Enzymproduktion im Glucose-Medium (Tabelle 2). Bei der Verwendung von Laminarin und Cellulose als C-Quelle wurde eine verminderte Enzymaktivitär festgestellt.

Tabelle 1

Abbau von Substraten durch Exoenzymaktivität

\begin{tabular}{lcccc}
\hline Substrat & Tributyrin & Chitin & Stärke & Protein \\
\hline & 1,0 & 1,6 & 4,2 & 2,1 \\
& 0,8 & 1,7 & 4,3 & 2,0 \\
& 0,9 & 1,7 & 4,1 & 2,3 \\
& - & - & 4,0 & 2,1 \\
Mittelwert & - & - & 4,1 & 2,3 \\
Standardabweichung & 0,9 & 1,7 & 4,1 & 2,2 \\
& 0,10 & 0,06 & 0,11 & 0,13 \\
\hline
\end{tabular}

Angegeben sind Durchmesser von Abbauzonen in $\mathrm{cm}$.

Tábelle 2

Enzym-Induktionswersuch

\begin{tabular}{|c|c|c|}
\hline \multirow[b]{2}{*}{ Spez. C-Quelle } & \multicolumn{2}{|c|}{ Enzymaktivität in: } \\
\hline & Glucosemedium & Medium mit spez. C-Quelle \\
\hline Laminarin & 51,1 & 47,7 \\
\hline Stärke & 2,5 & 3,89 \\
\hline Cellulose & 0,72 & 0,39 \\
\hline Chitin & 1,6 & 3,6 \\
\hline
\end{tabular}
$\mu \mathrm{mol} / \mathrm{l}$.

Angegeben sind Konzentrationen freigesetzter Glucose- bzw. NAc-Glucosæminmoleküle in

\section{Diskussion}

In der vorliegenden Arbeit wird gezeigt, über welche Infektionswege der Hyperparasit die Uredo- und Teleutosporen des Bohnenrostpilzes U. appendiculatus var. appendiculatus befällt und welche Mittel ihm zum Abbau der Sporen zur Verfügung stehen. Die Untersuchung von Zelloberflächen mit Gold-markierten Lektinen sollte zeigen, ob für die Penetration des Hyperparasiten determinierte Stellen auf den Sporen nachweisbar sind, und ob Zuckermoleküle eine Rolle bei der Erkennung spielen.

Bereits veröffentlichte Untersuchungen von Verticillium auf Uredosporen verschiedener Rostpilze (Allen 1980, Hänssler 1981, MeNDGEN 1981 und SPENCER 1981) haben die Penetration der Sporenwände, den Abbau des Sporeninhaltes und die Deformation der Uredosporen gezeigt. Während jedoch beim Gelbrost 
ein kompletter Wandabbau der infizierten Uredosporen beschrieben wurde (MENDGEN 1979), kollabierten die Uredosporen des Bohnenrostes offensichtlich als Folge des Herauslösens von Chitin aus der Sporenwand und dem Auflösen der Zellinhalte. Bei der hier erstmalig beschriebenen Infektion von Teleutosporen wird über der Penetrationsstelle ein Appressorium ausgeprägt. Sporeninhalt agglutiniert und wird im weiteren Verlauf der Infektion abgebaut. Im Vergleich zu den Uredosporen wird dem Hyperparasiten bei dem Befall der Teleutosporen der Zugang zum Sporeninhalt erschwert: Keimporen werden nicht geöffnet, die Sporenwände sind verstärkt. Die bei Uredosporen beobachtete Deformation unterbleibt. Die Infektion von. Teleutosporen ist von besonderer Bedeutung, da die Teleutospore die Lebensform des Bohnenrostpilzes darstellt, die ein Überwintern des Rostpilzes sichert. Sie ist der Ausgangspunkt für den neuen Lebenszyklus im Frühjahr. Zum Nachweis der für den Abbau von Rostsporen wahrscheinlich notwendigen Enzyme kamen u. a. Verfahren von Dомsсн (DомscH 1960) zur Anwendung, der bereits Cellulose-, Chitin- und Eiweißabbau bei $V$. lecanii konstatierte. Unsere Untersuchungen erweitern das Wissen über das Spektrum an verwertbaren Substraten, indem der Abbau von Stärke, Tributy rin und Laminarin nachgewiesen wurde und geben eine Vorstellung, wie sich der fortschreitende Abbau von Rostsporen abspielen könnte: Chitin ist ein in den Sporenwänden des Bohnenrostpilzes nachgewiesenes Strukturpolymer (TrOCHA und DALY 1974). Wenn der Hyperparasit über die Rostspore wächst, dann erhält er vermutlich Chitinabbauprodukte in Folge seiner Enzymausscheidungen. Diese wiederum induzieren eine vermehrte Produktion chitinolytischer Enzyme. Dieser sich selbst verstärkende Prozeß führt letztendlich zum Abbau der Rostsporen. Ebenso führt das Vorhandensein von Stärke im Sporenplasma zu einer Aktivierung der Ausscheidung von stärkeabbauenden Enzymen, und somit zu einer weiteren Beschleunigung des Abbauprozesses. Die von LeINHOS und BUCHENAUER 1984 beschriebene Wirkung zellfreier Kulturfiltrate nach präinfektioneller Blattapplikation gegen den Befall von Hafer mit Puccinia coronata kann dagegen nicht auf die Aktivität von Enzymen zurückgeführt werden. Hier scheinen noch weitere, nicht vollständig aufgeklärte Mechanismen zur Rostbefallsminderung beizutragen. Obwohl Zuckermoleküle sowohl auf der Oberfläche von Uredound Teleutosporen, als auch auf den Hyphen des Hyperparasiten nachweisbar waren, konnte keine Korrelation zwischen dem Vorhandensein der Zucker und den vom Hyperparasiten gewählten Infektionsstellen festgestellt werden. Möglicherweise haben diese Moleküle jedoch eine Funktion als unspezifische Induktoren von sporenwandabbauenden Enzymen.

\section{Literaturverzeichnis}

AlLEN, D. J., 1982: Verticillium lecanii on the bean rust fungus Uromyces appendiculatus. Trans. Br. mycol. Soc. 79 (2), 362-364.

Davis, B. D., and E. S. Miniolo, 1950: Mutants of E. coli requiring methionene or vitamine B 12. J. Bact. 60, 17-28.

Domsch, K. H., 1960: Das Pilzspektrum einer Bodenprobe III. Nachweis der Einzelpilze. Arch. Mikrobiol. 35, 310-339. 
Elad, Y., I. Chet, P. Boyle, and Y. HenIs, 1983: Parasitism of Trichoderma species on Rbizoctonia solani and Sclerotium rolfsii - scanning electron microscopy and fluorescene microscopy. Phytopathology $73(1), 85-88$.

FRENS, G., 1973: Controlled nucleation for the regulation of particle size in monodisperse gold suspensions. Nature Phys. Sci. 241, 20-22.

Grabski, G. C., und K. MENDGEN, 1985: Einsatz von V. lecanii als biologisches Schädlingsbekämpfungsmittel im Feld und im Gewächshaus. Phytopath. Z. 113, 243-251.

Hänssler, G., M. Hermanns, und H. R. Reisener, 1982: Elektronenmikroskopische Beobachtung der Interaktion zwischen Uredosporen von Puccinia graminis var. tritici und Verticillium lecanii. Phtopath. Z. 103, 139-148.

Hankin, L., and S. L. AnAgnostakis, 1975: The use of solid media for detection of enzyme production by fungi. Mycologia 67, 597-605.

HorisBerger, M., and J. ROSSET, 1977: Colloidal gold, a useful marker for transmission and scanning electron microscopy. J. Histochem. Cytochem. 25 (4), 295-305.

- -, 1979: Evaluation of colloidal gold as a cytochemical marker for transmission and scanning electron microscopy. Biol. Cell. 36, 253-260.

Leinhos, G., und H. BuCHENAuer, 1984: Untersuchungen zu Antagonismus und Hyperparasitismus einiger ausgewählter Pilze gegenüber Getreiderostpilzen. Mitt. Biol. BundAnst. f. Land- und Forstw. 223, 255.

MeNDGEN, K., and R. CASPER, 1979: Quantitative serological estimation of a hyperparasite: Detection of Verticillium lecanii in yellow rust infected wheat leaves by ELISA. Phytopath. Z. 94, $89-91$.

- -, 1981: Growth of Verticillium lecanii in pustules of stripe rust Puccinia graminis. Phytopath. Z. 102, 301-309.

Mourey, A., and G. Kilbertus, 1976: Simple media containing stabilized tributyrin for demonstrating lipolytic bacteria in foods and soils. J. Appl. Bacteriol. 40, 47-51.

Reissig, J. L., J. L. Strominger, and L. F. Leloir, 1955: A modified colorimetric method for estimation of NAc-amino sugars. J. Biol. Chem. 217, 959-966.

ReYNolds, E. S., 1963: The use of lead citrate at high $\mathrm{pH}$ as an electron-opaque stain in electron microscopy. J. Cell Biol. 17, 208.

Sequeira, L., 1978: Lectins and their role in host-pathogen specifities. Ann. Rev. Phytopath. 16, $453-481$.

Spencer, D. M., and P. T. Atkey, 1981: Parasitic effects of Verticillium lecanii on two rust fungi. Trans. Br. mycol. Soc. 77 (3), 535-542.

Trocha, P., and J. M. Daly, 1974: Cell walls of germinating uredospores. II.: Carbohydrate polymers. Plant Physiol. 53, 527-532.

Adresse der Autoren: Dr. C. Grabski, SANDOZ AG, Agrarbiologische Versuchsstation Witterswil, CH 4108 Witterswil (Schweiz). Prof. Dr. K. MendGen, Universität Konstanz, Lehrstuhl für Phytopathologie, Postf. 5560, 7750 Konstanz (F.R.G.). 